\title{
Compensation of Thermally Induced Modal Distortions in Faraday Isolators
}

\author{
Efim Khazanov, Nikolay F. Andreev, Anatoly Mal'shakov, Oleg Palashov, Anatoly K. Poteomkin, Alexander Sergeev, \\ Andrey A. Shaykin, Victor Zelenogorsky, Igor A. Ivanov, Rupal Amin, Guido Mueller, David B. Tanner, and \\ David H. Reitze
}

\begin{abstract}
Two methods of compensation of thermal lensing in high-power terbium gallium garnet (TGG) Faraday isolators have been investigated in detail: compensation by means of an ordinary negative lens and compensation using FK51 Schott glass possessing a negative $d n / d T$. Key thermooptic constants for TGG crystals and FK51 glass were measured. We find that the contribution of the photoelastic effect to the total thermal lens cannot be neglected for either TGG or FK51. We define a figure of merit for compensating glass and show that for FK51, an ordinary negative lens with an optimal focus is more efficient, but requires physical repositioning of the lens for different laser powers. In contrast, the use of FK51 as a compensating element is passive and works at any laser power, but is less effective than simple telescopic compensation. The efficiency of adaptive compensation can be considerably enhanced by using a compensating glass with figure of merit more than 50 , a crystal with natural birefringence or gel.
\end{abstract}

Index Terms-Faraday effect, laser accessories, laser beam distortion, laser thermal factors, optical isolators, optical polarization, optical propagation in anisotropic media, thermal variables measurements.

\section{INTRODUCTION}

$\mathbf{R}$ ECENTLY, the average power of solid-state and fiber lasers has overcome the kilowatt barrier and continues to increase steadily [1], [2]. Therefore, the search for methods of suppressing thermal effects caused by laser absorption in bulk optical elements has become ever more topical. In particular, Faraday isolators (FIs) are strongly affected by thermal self-action, since the absorption in magnetooptical media is relatively high. A number of papers have been devoted to investigations of self-induced thermal effects in magnetooptical media [3]-[16]. The absorption of radiation in optical elements of FIs generates a temperature distribution that is nonuniform over a transverse cross section. This leads to three physical mechanisms affecting the laser radiation: thermal lensing; a nonuniform distribution of the angle of rotation of the polarization plane because of the temperature dependence

Manuscript received January 9, 2004; revised June 1, 2004. This work was supported in part by the National Science Foundation under Grant PHY0140110 and Grant PHY0244902.

E. Khazanov, N. F. Andreev, A. Mal'shakov, O. Palashov, A. K. Poteomkin, A. Sergeev, A. A. Shaykin, and V. Zelenogorsky are with the Institute of Applied Physics, Russian Academy of Sciences, Nizhny Novgorod 603600, Russia (e-mail: khazanov@appl.sci-nnov.ru).

I. A. Ivanov is with The Research Institute of Materials Science and Technology, Zelenograd, Russia (e-mail: iia@zelmail.ru).

R. Amin, G. Mueller, D. B. Tanner, and D. H. Reitze are with the University of Florida, Gainesville, FL 32611-8440 USA (e-mail: reitze@ phys.ufl.edu).

Digital Object Identifier 10.1109/JQE.2004.834766

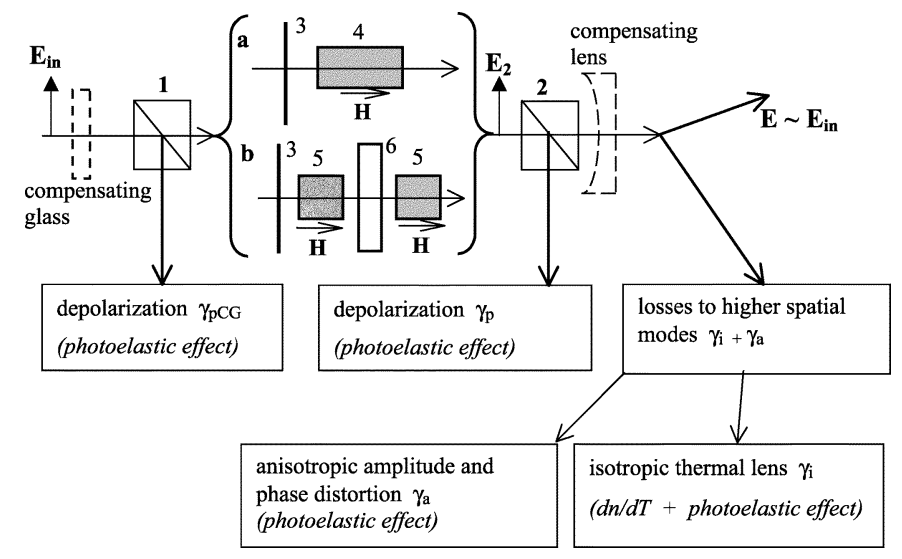

Fig. 1. Overview of power loss mechanisms in the spatial polarization mode after passage of a laser beam through (a) a traditional FI and (b) a birefringencecompensated FI for use at high average powers. Optical components are indicated numerically: 1, 2-polarizers; 3-half-wave plate; 4- $45^{\circ}$ Faraday rotator; $5-22.5^{\circ}$ Faraday rotator; $6-67.5^{\circ}$ reciprocal rotator.

of the Verdet constant; and the simultaneous appearance of circular birefringence (Faraday effect) and linear birefringence due to the photoelastic effect. The latter two mechanisms alter the polarization state of radiation transmitted through the FI, deteriorating the isolation ratio. This was studied in detail in [6], where it was shown that the photoelastic effect makes the greatest contribution to the depolarization. In [5], novel designs of FIs were suggested and theoretically justified, in which the deterioration in the isolation ratio was considerably improved. Further experiments [8], [12] confirmed the high efficiency of the novel designs. The influence of laser beam shape on all these parameters was considered in [10]. The dependence of the depolarization ratio in differing FI designs on orientation of magnetooptic crystal was thoroughly investigated in [14]. Another method for compensating depolarization in the FI was suggested and studied in [15], based on the use of crystalline quartz cut along its optical axis and placed inside a telescope. Taking all of these prior investigations as a whole, the most efficient and convenient design is that of a reciprocal quartz rotator [Fig. 1(b)].

The isolation ratio is generally the primary but not the only parameter of interest in measuring the performance of an FI. In addition, beam distortions are also induced in these devices through thermal lensing and other mechanisms. In some applications, these characteristics may be as important as the isolation. An example is an FI in a laser interferometer for gravi- 
tational wave detection [17] or a Faraday mirror inside a laser oscillator [13], [16], [18] or regenerative amplifier [18].

In the following sections, we consider the performance of two different FI designs-the traditional single element design [Fig. 1(a)] and the birefringence-compensated design [Fig. 1(b)] [5]. The latter type employs, instead of a single $45^{\circ}$ Faraday rotator, two $22.5^{\circ}$ Faraday rotators and a reciprocal polarization rotator placed between them, which rotates the polarization at $67.5^{\circ}$. Let us assume that an incident beam $\boldsymbol{E}_{\text {in }}$ has horizontal polarization, Gaussian intensity profile with waist $r_{0}$ and flat wavefront, i.e., the complex amplitude of the field is given in the form

$$
\boldsymbol{E}_{\text {in }}=\boldsymbol{x}_{0} E_{0} \exp \left(\frac{-r^{2}}{2 r_{0}^{2}}\right) \exp [i(k z-\omega t)]
$$

where $x_{0}$ is a unit vector directed along the $x$ axis, $r$ is the polar radius, $k$ is the wavenumber, and $\omega$ is the frequency of the laser field. We wish to compare the total power losses in the spatial polarization mode (1) during the first pass (from left to right) of the beam through each isolator design. In both designs, in the absence of thermal effects after the first pass, the beam retains its horizontal polarization and passes through a polarizer 2 (while during the return pass the polarization is altered to vertical and the beam is reflected by a polarizer 1). Because of polarization distortions in the magnetooptical medium, part of the radiation will be reflected by the polarizer 2 after the first pass. The corresponding power loss $\gamma_{p}$ (henceforth, called polarization loss) is determined as a ratio of radiation power reflected from polarizer 2 to radiation power incident on polarizer 2

$$
\gamma_{p}=\frac{\int_{0}^{2 \pi} d \varphi \int_{0}^{\infty}\left|\boldsymbol{E}_{2} \cdot \boldsymbol{y}_{0}\right|^{2} r d r}{\int_{0}^{2 \pi} d \varphi \int_{0}^{\infty}\left|\boldsymbol{E}_{2}\right|^{2} r d r}
$$

where $\boldsymbol{y}_{0}$ is a unit vector directed along the $y$ axis, $\varphi$ is the azimuthal angle, and $\boldsymbol{E}_{2}$ is the complex amplitude of the field before the polarizer 2. Here (and later), we assume that the clear aperture of FI is such that the aperture losses can be ignored and the integration over $r$ can be extended to infinity. The component of the field transmitted through the polarizer, $\boldsymbol{E}_{2, x}$ is linearly polarized (polarizer 2 is assumed to be ideal), but its transverse structure now differs from the incident Gaussian beam $\boldsymbol{E}_{\text {in }}$ because of the spatially dependent amplitude and phase distortions introduced during propagation through the nonuniformly heated magnetooptic medium and the polarizer. We define a second quantity, $\gamma_{\mathrm{s}}$, as the difference from unity of projection of the laser field $E_{2, x}$ on the ideal Gaussian field (1)

$$
\gamma_{s}=1-\frac{\left|\int_{0}^{2 \pi} d \varphi \int_{0}^{\infty} E_{2, x} E_{\mathrm{in}}^{*} r d r\right|^{2}}{\int_{0}^{2 \pi} d \varphi \int_{0}^{\infty}\left|E_{2, x}\right|^{2} r d r \cdot \int_{0}^{2 \pi} d \varphi \int_{0}^{\infty}\left|E_{\mathrm{in}}\right|^{2} r d r} .
$$

Physically, this quantity represents the "effective" power loss from the Gaussian $\left(\mathrm{TEM}_{00}\right)$ beam caused by the introduction of higher order spatial modes transmitted through the polarizer. In the absence of thermal effects, $\gamma_{\mathrm{s}}=0$. Therefore, the total power loss in the $\mathrm{TEM}_{00}$ spatial polarization mode $\gamma_{t}$ during forward propagation through the FI is

$$
1-\gamma_{t}=\left(1-\gamma_{p}\right)\left(1-\gamma_{s}\right)
$$

It has been shown [10], [11] that two different effects contribute to $\gamma_{s}$ : an isotropic thermal lens and anisotropic distortions caused by the photoelastic effect. For small distortions $\left(\gamma_{s} \ll 1\right), \gamma_{s}$ is a sum of two corresponding components: anisotropic amplitude-phase losses $\gamma_{a}$ and isotropic phase losses $\gamma_{i}$ [10], [11]. Therefore, the total power loss in the spatial polarization mode $\gamma_{t}$ during propagation is (for $\gamma_{p} \ll 1$ )

$$
\gamma_{t}=\gamma_{p}+\gamma_{a}+\gamma_{i} .
$$

The first two components are related to depolarization caused by the photoelastic effect, whereas the latter component represents pure optical path (phase) distortions induced by the isotropic thermal lens. These losses are indicated schematically in Fig. 1. Note that a contribution to the isotropic thermal lens is made by both the temperature dependence of refractive index and the "isotropic" part of the photoelastic effect (see below). It has been shown previously [10], [11] that $\gamma_{\mathrm{i}}$ is the same for all FI designs.

The temperature distribution in the optical element and, consequently, the distribution of phase of an aberrated laser beam are close to parabolic. Therefore, the majority of the phase distortions can be compensated by means of an ordinary lens or a telescope (shown as a dashed line in Fig. 1), which introduces additional curvature in the wavefront. Throughout the text, we shall, henceforth, call this method of compensation "telescopic compensation," indicating corresponding losses by subscript "TC." Obviously, the isotropic thermal lens is not totally compensated, since the ordinary lens can compensate only for parabolic phase, but the thermal lens has a more complex profile. For small distortions, it has been shown that the isotropic losses introduced by the thermal lens can be reduced approximately by a factor of 15 , provided the position and focus of the compensating lens is chosen optimally [10], [11]

$$
\gamma_{i \mathrm{TC}} \approx \frac{\gamma_{i \mathrm{NC}}}{15}
$$

Here, and further, the subscript "NC" indicates that there is no compensation present and the subscript $i$ refers to the isotropic component of depolarization.

Alternatively, an adaptive method for compensating the thermal lens was suggested and experimentally studied [19], [20]. Essentially, it consists of a compensating glass (CG) placed before (or after) polarizer 1 (e.g., Schott glass FK51; indicated as the dashed line in Fig. 1). The parameters of the CG are chosen such that the thermal lens has the same amplitude and shape as in FI but, at the same time, is negative (in many magnetooptic materials it is positive). If we do not take into 
account the propagation of the beam between CG and FI, the isotropic losses are totally compensated

$$
\gamma_{i \mathrm{AC}}=0
$$

Here, and later, the subscript "AC" denotes the adaptive compensation. It has been shown that the influence of propagation can be insignificant for reasonable adaptive compensation geometries [19].

The adaptive method has two advantages over the telescopic compensation: there is no need for adjustment when laser power is changed, and the accuracy of isotropic compensation is higher. However, a disadvantage of the adaptive method is that the photoelastic effect in the CG leads to additional distortions and, consequently, to losses in the spatial polarization mode. By analogy with losses introduced into FI, these losses can be subdivided into polarization losses $\gamma_{p C G}$, (power reflected by polarizer 1), and anisotropic amplitude-phase losses $\gamma_{a \mathrm{CG}}$ (see Fig. 1).

As we show in this paper, the above two compensation methods can compensate only for $\gamma_{i}$. The telescopic method is less efficient but does not lead to additional losses $\gamma_{p}$ and $\gamma_{a}$. The adaptive method totally compensates for $\gamma_{i}$ (if we ignore propagation), but increases losses $\gamma_{p}$ and $\gamma_{a}$ because of the photoelastic effect in the CG. This effect was not considered by Mueller et al. [19]. In Section II, we present a detailed comparison of the two compensation methods-telescopic and adaptive-considering all thermal effects both in the magnetooptic crystal and in compensating glass. Based on these results, we determine physical constants and define a figure of merit for the compensating glass that predicts the efficiency of the adaptive method. In Section III, we report on the results of measurement of key thermooptical constants for TGG crystal and FK51 glass. We also describe experimental results of compensation of the thermal lens in FI with a quartz rotator [Fig. 1(b)] using FK51 glass. In Section IV, our results will be discussed and our conclusions are summarized in Section V.

\section{EFFICIENCY OF MODE DISTORTION COMPENSATION}

The calculation of total losses without compensation $\gamma_{t \mathrm{NC}}$ and with telescopic compensation $\gamma_{t \mathrm{TC}}$ have been previously [10], [11] derived analytically. Here we shall calculate, by analogy with those papers, the total loss in case of adaptive compensation $\gamma_{t \mathrm{AC}}$ and shall compare the results with $\gamma_{t \mathrm{NC}}$ and $\gamma_{t \mathrm{TC}}$. We assume that the Rayleigh range of the beam is much greater than the length of FI, even taking into account induced distortions, i.e., diffractive propagation effects can be totally neglected. It is seen from (2) to (4) that for determining the total loss $\gamma_{t A C}$, it is enough to find the field before a second polarizer $E_{2}$. This value can be found by applying the formalism of the Jones polarization matrices

$$
\begin{aligned}
\boldsymbol{E}_{2}= & \mathbf{F}\left(\delta_{\mathrm{c}}=\frac{\pi}{2}, \delta_{1}, \mathrm{~L}\right) \mathbf{L}_{2}\left(\frac{3 \pi}{8}\right) \mathbf{P}_{x} \mathbf{G}\left(\delta_{\mathrm{CG}}\right) \boldsymbol{E}_{\mathrm{in}} \\
\boldsymbol{E}_{2}= & \mathbf{F}\left(\delta_{\mathrm{c}}=\frac{\pi}{4}, \frac{\delta_{1}}{2}, \frac{\mathrm{L}}{2}\right) \mathbf{R}\left(\frac{-3 \pi}{8}\right)\left|\mathbf{F}\left(\delta_{\mathrm{c}}=\frac{\pi}{4}, \frac{\delta_{1}}{2}, \frac{\mathrm{L}}{2}\right)\right| \\
& \times \mathbf{L}_{2}\left(\frac{\pi}{16}\right) \mathbf{P}_{x} \mathbf{G}\left(\delta_{\mathrm{CG}}\right) \boldsymbol{E}_{\mathrm{in}} .
\end{aligned}
$$

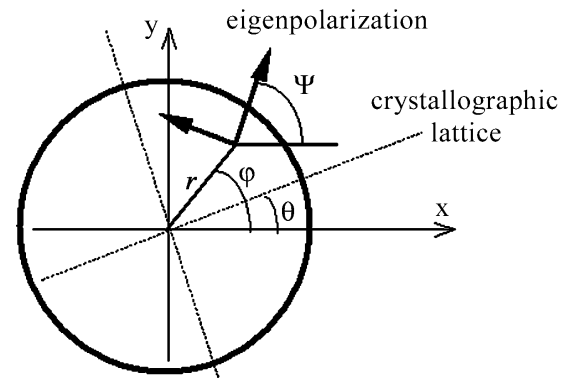

Fig. 2. Crystal cross section showing the crystallographic axes and eigenpolarizations for the calculations described in the text.

Here, and later, letters "a" and "b" correspond to formulas for FI in Fig. 1(a) and (b). F, $\mathbf{L}_{2}, \mathbf{R}, \mathbf{P}_{x}$, and $\mathbf{G}$ denote the Jones matrices for the Faraday rotator, $\lambda / 2$ waveplate, quartz rotator, polarizer, and compensating glass, respectively, $\delta_{l}$ is the phase difference between linear eigenpolarizations along the entire length of the magnetooptical medium $L$; and $\delta_{c}$ is the phase difference between circular eigenpolarizations. The matrices for the rotator, $\lambda / 2$ plate and polarizer are well known

$$
\begin{aligned}
R\left(\beta_{R}\right) & =\left(\begin{array}{cc}
\cos \beta_{R} & \sin \beta_{R} \\
-\sin \beta_{R} & \cos \beta_{R}
\end{array}\right) \\
L_{2}\left(\beta_{L}\right) & =\left(\begin{array}{cc}
\cos 2 \beta_{L} & \sin 2 \beta_{L} \\
\sin 2 \beta_{L} & -\cos 2 \beta_{L}
\end{array}\right) \\
P_{x} & =\left(\begin{array}{ll}
1 & 0 \\
0 & 0
\end{array}\right)
\end{aligned}
$$

where $\beta_{R, L}$ are the rotation angles. Bearing in mind the linear birefringence, a Faraday rotator that rotates the polarization plane by an angle $\delta_{\mathrm{c}} / 2$ can be described by the following Jones matrix [21], [22]:

$$
\begin{aligned}
F\left(\delta_{c}, \delta_{l}, L, \Psi\right)= & \exp (i k L n) \cdot \sin \frac{\delta}{2} \\
& \cdot\left(\begin{array}{cc}
\cot \frac{\delta}{2}-i \frac{\delta_{l}}{\delta} \cos 2 \Psi & -\frac{\delta_{c}}{\delta}-i \frac{\delta_{l}}{\delta} \sin 2 \Psi \\
\frac{\delta_{c}}{\delta}-i \frac{\delta_{l}}{\delta} \sin 2 \Psi & \cot \frac{\delta}{2}+i \frac{\delta_{l}}{\delta} \cos 2 \Psi
\end{array}\right)
\end{aligned}
$$

where

$$
\delta^{2}=\delta_{l}^{2}+\delta_{c}^{2}
$$

$\Psi$ is the angle of inclination of the linear eigenpolarization relative to the $x$ axis (see Fig. 2), $n$ is the refractive index averaged for two eigenpolarizations. We shall consider here only cylindrical optical elements and only two most common orientations- [001] (with an angle $\theta$ between the crystallographic axis and the $x$ axis) and [111]. Note that for the traditional FI design [Fig. 1(a)], the best orientation is [001], whereas [111] is the optimal crystal orientation for the birefringence compensated design [Fig. 1(b)] [14].

The value of $n(r)$ is determined by the temperature distribution $T(r)$. The expression for $n(r)$ can be found, e.g., in [23]

$$
n(r)=n(0)+[T(r)-T(0)] P
$$


where $P$ for the crystal orientations under consideration is

$$
\begin{aligned}
P_{[001]} & =\frac{d n}{d T}-\left(\frac{1}{L} \frac{d L}{d T}\right) \frac{n_{0}^{3}}{4} \frac{1+\nu}{1-\nu} \cdot\left(p_{11}+p_{12}\right) \\
P_{[111]} & =P_{[001]}-Q \frac{\xi-1}{3} \\
Q & =\left(\frac{1}{L} \frac{d L}{d T}\right) \frac{n_{0}^{3}}{4} \frac{1+\nu}{1-\nu} \cdot\left(p_{11}-p_{12}\right) \\
\xi & =\frac{2 p_{44}}{p_{11}-p_{12}}
\end{aligned}
$$

and $n_{0}, \nu,(1 / L)(d L / d T), p_{i, j}$ are the nonexcited ("cold") refractive index, Poisson's ratio, thermal expansion coefficient, and photoelastic coefficients of the magnetooptic medium, respectively.

The exponential phase factor in (8) does not affect the polarization distortions and represents an isotropic thermal lens. The temperature dependence of the refractive index and the "isotropic" part of the photoelastic effect contribute to this lens (see two corresponding components in (11) for $P_{[001]}$ ). In [24] and [25], it was shown that the contribution of the thermal expansion along the $z$ direction of the medium to the thermal lens is negligibly small, as compared to the temperature dependence of the refractive index. Therefore, we assume that length $L$ is independent of temperature.

The values of $\delta_{l}$ and $\Psi$ are determined by the photoelastic effect and depend on the temperature gradient $d T(r) / d r$ [23], [26]

$$
\begin{aligned}
& \left\{\begin{array}{l}
\delta_{l}(r, \varphi)=\frac{4 \pi L}{\lambda} Q\left[\frac{1+\xi^{2} \tan ^{2}(2 \varphi-2 \theta)}{1+\tan ^{2}(2 \varphi-2 \theta)}\right]^{\frac{1}{2}} \frac{1}{r^{2}} \int_{0}^{r} r^{2} \frac{d T}{d r} d r \\
\tan (2 \Psi-2 \theta)=\xi \tan (2 \varphi-2 \theta), \quad \text { for }[001]
\end{array}\right. \\
& \left\{\begin{array}{l}
\delta_{l}(r, \varphi)=\frac{4 \pi L}{\lambda} Q\left[\frac{1+2 \xi}{3}\right] \frac{1}{r^{2}} \int_{0}^{r} r^{2} \frac{d T}{d r} d r \\
\Psi=\varphi, \quad \text { for }[111]
\end{array}\right.
\end{aligned}
$$

where $\lambda$ is a wavelength. As seen, the expressions for $\delta_{l}$ and $\Psi$ for the [001] orientation transform into expressions for $\delta_{l}$ and $\Psi$ for the [111] orientation, when $\xi$ is substituted by unity and $Q$-by $Q(1+2 \xi) / 3$. Therefore, we shall, henceforth, only give expressions only for the [001] orientation, bearing in mind that corresponding formulas for the [111] orientation can be obtained by these substitutions. We also drop the [111], [001] subscripts from $P, Q$ when referring to TGG.

The temperature distribution $T(r)$ can be easily found from the thermal conductivity equation for an infinitely long cylinder

$$
T(r)-T(0)=-\frac{\alpha P_{0}}{2 \pi \kappa} \cdot \int_{0}^{r} \frac{1-\exp \left(\frac{-r^{2}}{r_{0}^{2}}\right)}{r} d r
$$

where $\alpha$ and $\kappa$ are the absorption coefficient and the thermal conductivity of the magnetooptical medium, and $P_{0}$ is the laser radiation power.

Thus, (9)-(16) fully determine the matrix of the Faraday rotator (8). From a physical point of view, the compensating glass is equivalent to a Faraday element without a magnetic field present. Consequently, its matrix $\boldsymbol{G}$ is simply matrix $\boldsymbol{F}$ at $\delta_{c}=0$. All constants of the magnetooptical medium $(L$, $\left.n_{0}, \nu, p_{i, j}, \alpha, \kappa, P, Q\right)$ must be replaced with the appropriate constants of compensating glass $\left(L_{\mathrm{CG}}, n_{0 \mathrm{CG}}, \nu_{\mathrm{CG}}, p_{i, j \mathrm{CG}}\right.$, $\left.\alpha_{\mathrm{CG}}, \kappa_{\mathrm{CG}}, P_{\mathrm{CG}}, Q_{\mathrm{CG}}\right)$. Note that $\xi_{\mathrm{CG}}$ for any glass is equal to unity. Therefore, substitution of matrices $F$ and $G$, and (7) into (6), and then substitution of the result into (2)-(4), yield

$$
\begin{gathered}
\gamma_{t \mathrm{AC}}=\gamma_{p \mathrm{AC}}+\gamma_{a \mathrm{AC}}+\gamma_{i \mathrm{AC}} \\
\left\{\begin{array}{c}
\gamma_{p \mathrm{AC}}=\frac{A_{1}}{\pi^{2}} \cdot p^{2} \xi^{2}+\frac{A_{1}}{8} \cdot p_{\mathrm{CG}}^{2} \\
\gamma_{a \mathrm{AC}}=\frac{A_{1}}{\pi^{2}} p^{2}+\frac{A_{1}}{8} p_{\mathrm{CG}}^{2}+\frac{A_{1} \xi}{\pi \sqrt{8}} p \cdot p_{\mathrm{CG}} \\
\gamma_{i \mathrm{AC}}=\frac{A_{3}}{4}\left(p_{i}+p_{i \mathrm{CG}}\right)^{2}
\end{array}\right. \\
\left\{\begin{array}{cc}
\gamma_{p \mathrm{AC}}= & \frac{A_{1}}{\pi^{2}}(2-\sqrt{2}) p^{2}+\frac{A_{1}}{8} p_{\mathrm{CG}}^{2} \\
\gamma_{a \mathrm{AC}}= & \frac{A_{1}(2-\sqrt{2})}{\pi^{2}} \xi^{2} p^{2}+\frac{A_{1}}{8} p_{\mathrm{CG}}^{2} \\
& +\frac{A_{1}(\sqrt{2}+\xi-1)}{\pi \sqrt{8}} p p_{\mathrm{CG}} \\
\gamma_{i \mathrm{AC}}= & \frac{A_{3}}{4}\left(p_{i}+p_{i \mathrm{CG}}\right)^{2}
\end{array}\right.
\end{gathered}
$$

where we have defined the following quantities and constants:

$$
\begin{aligned}
A_{1} & =\int_{0}^{\infty}\left(\frac{1}{y}-\frac{\exp (-y)}{y}-1\right)^{2} \frac{d y}{\exp (y)} \cong 0.137 \\
A_{3} & =\int_{0}^{\infty} f^{2}(y) \exp (-y) d y-\left[\int_{0}^{\infty} f(y) \exp (-y) d y\right]^{2} \\
& \cong 0.268 \\
f(y) & =\int_{0}^{y} \frac{1-\exp \left(-y^{\prime}\right)}{y^{\prime}} d y^{\prime} \\
p_{i} & =\frac{L}{\lambda} \frac{\alpha P}{\kappa} P_{0} \quad p=\frac{L}{\lambda} \frac{\alpha Q}{\kappa} P_{0} \\
p_{i \mathrm{CG}} & =\frac{L_{\mathrm{CG}}}{\lambda} \frac{\alpha_{\mathrm{CG}} P_{\mathrm{CG}}}{\kappa_{\mathrm{CG}}} P_{0} \\
p_{\mathrm{CG}} q & =\frac{L_{\mathrm{CG}}}{\lambda} \frac{\alpha_{\mathrm{CG}} Q_{\mathrm{CG}}}{\kappa_{\mathrm{CG}}} P_{0} .
\end{aligned}
$$

Analytic expressions (17) and (18) are obtained for the case of weak distortions, i.e., they are valid at $\gamma \ll 1$. In our calculations, we assumed that the angle $\theta$ has its optimum value [10], [11]

$$
\begin{aligned}
& \theta_{\text {opt }}=-\frac{\pi}{8} \\
& \theta_{\text {opt }}=\frac{5 \pi}{16} .
\end{aligned}
$$

It is clear from (18) that for compensation of the isotropic part of losses $\gamma_{i}$ the following condition must be obeyed:

$$
p_{i \mathrm{CG}}=-p_{i}
$$

from which, taking into account (21), we obtain

$$
p_{\mathrm{CG}}=-\frac{P}{Q} \frac{Q_{\mathrm{CG}}}{P_{\mathrm{CG}}} p
$$

Thus, the key parameter of the compensating glass is the ratio $P_{\mathrm{CG}} / Q_{\mathrm{CG}}$, and the higher this ratio, the better the glass. Specifically, if this ratio is much more than that for the magnetooptical crystal, then losses associated with anisotropy thermally induced in the glass can be ignored. 
For comparison, we give expressions from [10] and [11] for the total loss without compensation

$$
\begin{gathered}
\gamma_{t \mathrm{NC}}=\gamma_{p \mathrm{NC}}+\gamma_{a \mathrm{NC}}+\gamma_{i \mathrm{NC}} \\
\left\{\begin{array}{l}
\gamma_{p \mathrm{NC}}=p^{2} \frac{A_{1}}{\pi^{2}} \xi^{2} \quad \gamma_{a \mathrm{NC}}=p^{2} \frac{A_{1}}{\pi^{2}} \\
\gamma_{i \mathrm{NC}}=\frac{A_{3}}{4} p_{i}^{2}
\end{array}\right. \\
\left\{\begin{array}{l}
\gamma_{p \mathrm{NC}}=\frac{p^{2} A_{1}}{\pi^{2}}(2-\sqrt{2}) \\
\gamma_{a \mathrm{NC}}=\frac{p^{2} A_{1}}{\pi^{2}}(2-\sqrt{2}) \xi^{2} \quad \gamma_{i \mathrm{NC}}=\frac{A_{3}}{4} p_{i}^{2} .
\end{array}\right.
\end{gathered}
$$

In the case of telescopic compensation, the first two components remain unchanged, whereas the third one is reduced approximately by a factor of 15 [10], [11]

$$
\begin{aligned}
\gamma_{t \mathrm{TC}}= & \gamma_{p \mathrm{NC}}+\gamma_{a \mathrm{NC}}+\frac{A_{4}}{A_{3}} \gamma_{i \mathrm{NC}} \\
A_{4}= & {\left[\int_{0}^{\infty}(f(y)-0.5 y)^{2} \exp (-y) d y\right] } \\
& -\left[\int_{0}^{\infty}(f(y)-0.5 y) \cdot \exp (-y) d y\right]^{2} \\
\cong & 0.0177 \cong 0.0660 A_{3} .
\end{aligned}
$$

(Note that in (27) for telescopic compensation ("TC"), we assume that the absorption in compensating lens is essentially zero, leading to negligible contributions for $\gamma_{t \mathrm{AC}}$ and $\gamma_{p \mathrm{AC}}$.) The comparison of (17) and (27) yields a condition, at which $\gamma_{t \text { AC }}<\gamma_{t \text { TC }}$, i.e., compensation with glass is more efficient than with a lens or a telescope

$$
\begin{gathered}
\left|\frac{P_{\mathrm{CG}}}{Q_{\mathrm{CG}}}\right|>\sqrt{\frac{A_{1}}{A_{4}}}\left(\sqrt{1+\frac{A_{1}}{2 A_{4}}\left[\frac{\xi Q}{\pi P}\right]^{2}}+\sqrt{\frac{A_{1}}{A_{4}}} \frac{\xi}{\pi \sqrt{2}}\left|\frac{Q}{P}\right|\right) \\
\left|\frac{P_{\mathrm{CG}}}{Q_{\mathrm{CG}}}\right|>\sqrt{\frac{A_{1}}{A_{4}}}\left(\sqrt{1+\frac{A_{1}}{2 A_{4}}\left[\frac{(\sqrt{2}+\xi-1) Q}{\pi P}\right]^{2}}\right. \\
\left.+\sqrt{\frac{A_{1}}{A_{4}}} \frac{(\sqrt{2}+\xi-1)}{\pi \sqrt{2}}\left|\frac{Q}{P}\right|\right) .
\end{gathered}
$$

Here, we took into account that $Q<0, Q_{\mathrm{CG}}<0, P_{\mathrm{CG}}<$ $0, P>0$ (see below). The ratio $P / Q$ has not been measured for the TGG crystal, which is most commonly used in FI; nor has it been found for the FK51 glass. Note that for laser $\mathrm{Nd}$ glasses, this ratio varies within a wide range: from 0.5 to 500 [27]. Therefore, the measurement of parameters $P$ and $Q$ for TGG and FK51 becomes important, and additional experiments are required to confirm the efficiency of the adaptive method for compensating the thermal lens.

\section{MEASuREMENT OF THERMOOPTICAL CONSTANTS OF TGG CRYSTAL AND FK51 GLASS}

From (17), (23)-(25), and (27), the energy losses in the spatial polarization mode $\gamma_{t}$ (both with and without compensation of the thermal lens) are totally determined by parameters $p$,

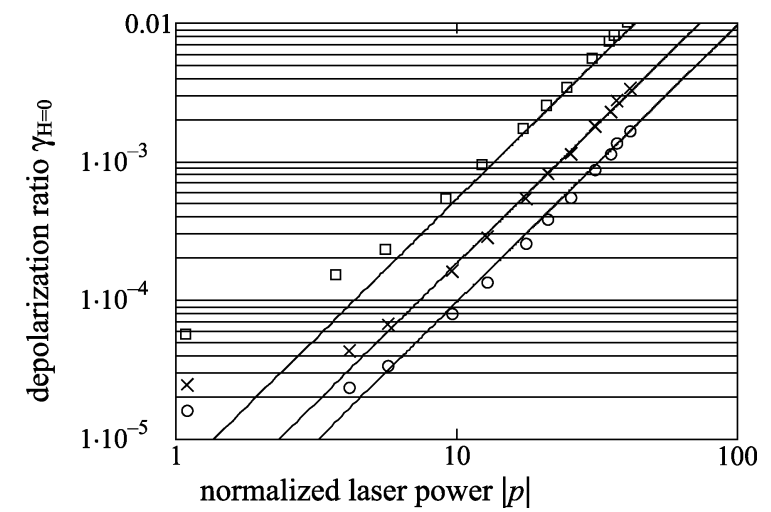

Fig. 3. Measurement of zero-field depolarization $\gamma_{\mathrm{H}=0}$ as a function of $p$ (see (21) for definition of $p$ ) for a 48-mm-long TGG crystal (manufactured by RIMST) at $\theta=0$ (squares) and $\theta=\pi / 4$ (circles) as well as for FK51 glass $67 \mathrm{~mm}$ in length (crosses). Theoretical curves are plotted according to (30) at $\alpha_{\mathrm{TGG}} Q_{\mathrm{TGG}}=1.2 \cdot 10^{-8} \mathrm{~K}^{-1} \cdot \mathrm{cm}^{-1}, \alpha_{\mathrm{FK} 51} Q_{\mathrm{FK} 51}=0.15 \cdot 10^{-8} \mathrm{~K}^{-1}$. $\mathrm{cm}^{-1}$.

$p_{\mathrm{i}}, \xi, Q_{\mathrm{CG}} / P_{\mathrm{CG}}$, and the smaller these parameters, the lower $\gamma_{t}$. Therefore, we must determine the following constants: $\kappa$, $\alpha, Q, P$, and $\xi$ for a magnetooptic medium (here we consider TGG only) and $Q_{\mathrm{CG}}, P_{\mathrm{CG}}$ for compensating glass (FK51). The thermal conductivity of TGG is $\kappa=7.4 \mathrm{~W} / \mathrm{km}$. The absorption coefficient of TGG $\alpha$ can vary significantly (up to a factor of four) from sample to sample [14], [19], [28], and the value of $\xi$ for TGG has been recently measured in [14] and [28]: $\xi=2.25 \pm 0.2$. More difficult is to measure the thermooptical constants $Q$ and $P$ (as well as $Q_{\mathrm{CG}}, P_{\mathrm{CG}}$ ), because of the difficulty in measuring their constituent constants, in particular, photoelastic coefficients $p_{i j}$. In Section III-A, we shall describe measurements of $Q$, carried out using a technique reported in [6]. In Section III-B, a scanning Hartmann sensor will be described, which we utilized for measuring $P$. The results of the measurements will be summarized in Section III-C.

\section{A. Measurement of Thermooptical Constant $Q$}

All known direct measurements of $Q$ to date have been made by means of an interferometric setup [29], [30]. The root-meansquare error of these measurements is typically $5 \%-15 \%$ [23]. Here, we employ a simple measurement technique [6] which provides the same accuracy. It consists of measuring the depolarization of high-power laser radiation propagating in an absorbing medium in the absence of a magnetic field $\gamma_{H=0}$ as a function of laser radiation power

$$
\gamma_{H=0}=p^{2} \frac{A_{1}}{8}\left(1+(\xi-1)^{2} \cos ^{2}(2 \theta)\right) .
$$

If all values, except $Q$ in the right-hand side of (30), are known, then by measuring $\gamma_{H=0}$, one can determine the constant $Q$, using it as a fitting parameter. We performed such measurements for FK51 Schott glass and for a [001] TGG crystal produced at the Research Institute of Materials Science and Technology, Zelenograd, Russia (RIMST). The measurement results are shown in Fig. 3. At small $p$, the depolarization $\gamma_{H=0}$ does not depend on power, since it is determined by the "cold" birefringence. At higher powers, the coincidence between experimental values and (30) is fairly good. 


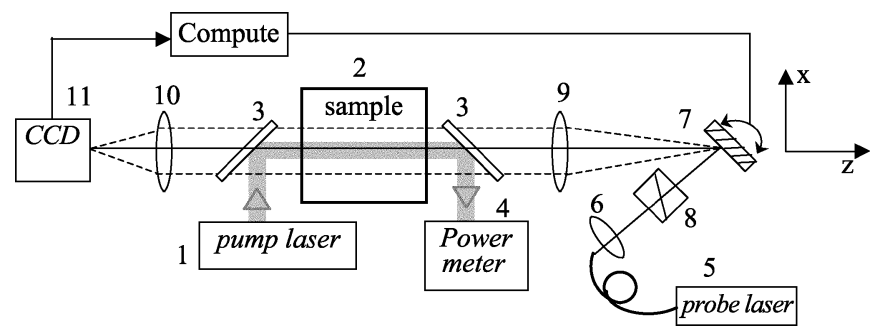

Fig. 4. Experimental schematic diagram for the scanning Hartmann sensor described in the text. Optical components are indicated numerically: 1 -pump laser; 2-sample; 3-mirror; 4-power meter; 5-probe laser; 6-collimating lens; 7-rotating mirror; 8-polarizer; 9, 10-lens; 11-CCD-camera.

The transmission and residual reflection of the anti-reflection coating were measured for this particular TGG crystal (length $48 \mathrm{~mm}$, diameter $9 \mathrm{~mm}$ ). Based on these data, we calculated the absorption. The transmission was measured at a wavelength of $1053 \mathrm{~nm}$ both for one and two passes of the radiation through the crystal. In the both cases, the value of the absorption was the same, $\alpha_{\mathrm{TGG}}=(4.8 \pm 0.4) \times 10^{-3} \mathrm{~cm}^{-1}$. Knowing $\alpha_{\mathrm{TGG}}$ from Fig. 3 we obtain the following value:

$$
Q_{\mathrm{TGG}}=-(2.6 \pm 0.4) \times 10^{-6} \mathrm{~K}^{-1} \text {. }
$$

The signs of $Q$ for TGG and FK51 (see below) was determined by the technique reported in [31]. The absorption of FK51 glass in the Schott catalog is $\alpha_{\mathrm{FK} 51}=2.4 \cdot 10^{-3} \mathrm{~cm}^{-1}$. Using $\alpha_{\mathrm{FK} 51}=2.4 \cdot 10^{-3} \mathrm{~cm}^{-1}$ from Fig. 3 we obtain

$$
Q_{\mathrm{FK} 51}=-0.63 \times 10^{-6} \mathrm{~K}^{-1} .
$$

The measured value of $\mathrm{Q}_{\mathrm{FK} 51}$ (32) is close to that in the Schott catalog, $Q_{\mathrm{FK} 51}=-(0.53-0.59) \cdot 10^{-6} \mathrm{~K}^{-1}$ for a wavelength of $589 \mathrm{~nm}$. For some glasses, it is known that $Q$ very weakly depends on wavelength [9]. However, keeping in mind that the accuracy of $\alpha_{\mathrm{FK} 51}=2.4 \cdot 10^{-3} \mathrm{~cm}^{-1}$ may be not very high, it is important to note that the value of the product $\alpha_{\mathrm{FK} 51} Q_{\mathrm{FK} 51}$ is measured with high accuracy

$$
\alpha_{\mathrm{FK} 51} Q_{\mathrm{FK} 51}=-(0.15 \pm 0.02) \times 10^{-8} \mathrm{~K}^{-1} \cdot \mathrm{cm}^{-1} .
$$

\section{B. Use of a Scanning Hartmann Sensor for Thermal Lens Measurements}

The detailed description of the operation of the scanning Hartmann sensor can be found elsewhere [28]. Here, we briefly describe its operation in measurements of thermal lensing. An optical schematic of the experiment is illustrated in Fig. 4.

The laser beam from a 40-W 1053-nm CW Nd:YLF singletransverse mode laser (indicated as " $l$ " in the figure) is used to heat the optic under test (2). Dichroic mirrors (3) are used to couple the beam into and out of the sample, with the laser power monitored using a power meter (4). An $850-\mathrm{nm}, \mathrm{CW}$ single-mode diode "probe" laser (5) with fiber output for mode cleaning is collimated by a lens $(6)$, with the output polarization set by a polarizer ( 8 ). This scheme provides a constant spatial structure of the probe beam. A rotating mirror (7), placed on the axis of a computer-controlled galvanometric scanner, changes the angular pointing of the beam. The solid line in Fig. 3 indicates the beam path in the midposition of the mirror (7), and the dotted lines-in two end positions. The rotational axis of the mirror (7) is located at the focal plane of a lens (9) with focal length $295 \mathrm{~mm}$ such that angular scanning of the mirror (7) results in a parallel movement of the beam along the diameter of the sample. In the presence of a thermal lens, the probe beam transmitted through the sample deviates from its initial direction by a small but measurable angle. This angle leads to a corresponding shift of the beam in the focal plane of a second $f=600 \mathrm{~mm}$ lens (10), which focuses the probe beam onto a CCD camera (11) located a distance $f$ from the lens. The signal from the CCD camera is sent to a computer, which determines and compares the coordinates of the beam's centroid position before and after heating the sample. By scanning the galvanometer, we are able to measure the differential optical path difference as a function of the heating power with a resolution of $\lambda / 500$.

In contrast to the traditional Hartmann sensor [20], [32], in which the entire aperture of the sample is measured simultaneously at many points, in this method one has to scan the beam over the sample aperture point by point. Although this procedure is time consuming, it avoids problems associated with overlapping of different beams which are frequently encountered in the traditional Hartmann sensor.

\section{Measurement of Thermooptical Constant P}

To eliminate depolarization of radiation from the probe laser in the sample, we used only $r$ - (the field along the polar radius) and $\varphi$ - (the field along the polar angle) polarizations. Measurements were made in the absence of a magnetic field for four cases: TGG with [111] orientation; TGG with [001] orientation at $\theta=0$; TGG with [001] orientation at $\theta=\pi / 4$; and FK51 glass. By analogy with Section II, it can be easily shown that in the first three cases the phase $\Delta(u)$ for $r$ - and $\varphi$-polarizations of the probe beam after a pass through a sample with the length $L_{\mathrm{s}}$ is described by the following expressions:

$$
\left\{\begin{array}{c}
\Delta_{r, \varphi}^{[111]}(u)=-\frac{L_{S}}{2 \lambda} \frac{\alpha P_{0}}{\kappa}\left(P_{[111]}\left[\int_{0}^{u} \frac{1-\exp (-t)}{t} d t\right]\right. \\
\left.\mp Q \frac{1+2 \xi}{3} \cdot \frac{u+\exp (-u)-1}{u}\right) \\
\Delta_{r, \varphi}^{[001]}(u, \theta=0)=-\frac{L_{S}}{2 \lambda} \frac{\alpha P_{0}}{\kappa}\left(P_{[001]}\left[\int_{0}^{u} \frac{1-\exp (-t)}{t} d t\right]\right. \\
\left.\mp Q \xi \frac{u+\exp (-u)-1}{u}\right) \\
\Delta_{r, \varphi}^{[001]}\left(u, \theta=\frac{\pi}{4}\right)=-\frac{L_{S}}{2 \lambda} \frac{\alpha P_{0}}{\kappa}\left(P_{[001]}\left[\int_{0}^{u} \frac{1-\exp (-t)}{t} d t\right]\right. \\
\left.\mp Q \frac{u+\exp (-u)-1}{u}\right) .
\end{array}\right.
$$

Here, $u=x^{2} / r_{0}^{2}$ is the scaled transverse coordinate. We use $x$ to denote the radial distance from the center of the crystal to reflect that our experiments scan the distortion in one dimension, see Fig. 3. For the glass, we can use any of the three expressions at $\xi=1$. Note that the distribution of the isotropic term (the first component) of the thermal lens is the same as the temperature distribution (16), whereas the anisotropic (the second component) part has a different shape. 

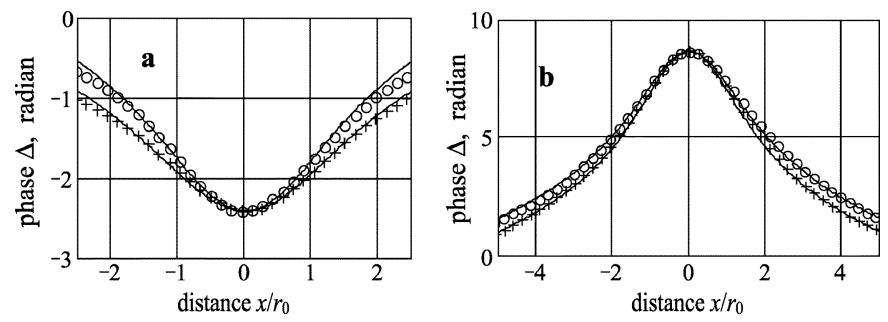

Fig. 5. Probe laser optical path difference after propagating through (a) a 48mm-long TGG crystal at $\theta=\pi / 4$ and (b) FK51 glass 67-mm length when heated by a $38-\mathrm{W}$ pump laser. Circles $-\varphi$-polarization; crosses- $r$-polarization. Theoretical curves are plotted according to (34) at $\alpha_{\mathrm{TGG}} P_{\mathrm{TGG}}=$ $1.5 \cdot 10^{-8} \mathrm{~K}^{-1} \cdot \mathrm{cm}^{-1}, \alpha_{\mathrm{FK} 51} P_{\mathrm{FK} 51}=-0.41 \cdot 10^{-8} \mathrm{~K}^{-1} \cdot \mathrm{cm}^{-1}$.

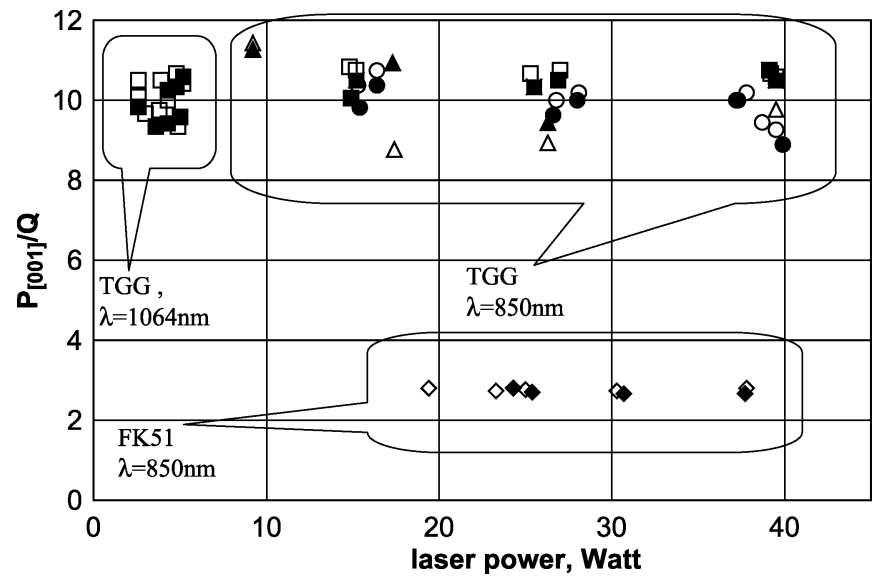

Fig. 6. The ratio $P_{[001]} / Q$ for FK51 glass (diamonds) and TGG crystals grown by EOT (circles), Litton (triangles), and RIMST (squares). Filled symbols correspond to $\varphi$-polarization and open symbols to $r$-polarization.

With the measurements of $\alpha$ and $Q$, all values except $P$ in (34) are known. $P$ was used as a fitting parameter. Measurements for FK51 Schott glass, two TGG crystals with the [001] orientation (from Electrooptic Technologies (EOT) and RIMST) and one TGG crystal with [111] orientation (from Litton) were carried out. The dependences $\Delta(r)$ for glass and the RIMST crystal are presented in Fig. 5. The figure clearly shows good coincidence between theoretical (34) and experimental curves, demonstrating that the measurement accuracy of $P$ was high and the sign of $Q$ was properly determined.

The measurements demonstrated the absence (within the experimental error) of the dependence of $P$ on power of the heating laser, and the absence of the dependence of $P_{[001]} / Q$ on a particular TGG sample (see Fig. 6). Knowing the absorption of the RIMST crystal $\alpha_{\mathrm{TGG}}=(4.8 \pm 0.4) 10^{-3} \mathrm{~cm}^{-1}$, from Fig. 5(a) we obtain the value of $P_{[001]}$

$$
P_{[001]}=(26 \pm 4) 10^{-6} \mathrm{~K}^{-1} \text {. }
$$

Using $\alpha_{\mathrm{FK} 51}=2.4 \cdot 10^{-3} \mathrm{~cm}^{-1}$, from Fig. 5(b) for the FK51 glass we obtain

$$
P_{\mathrm{FK} 51}=-1.7 \cdot 10^{-6} \mathrm{~K}^{-1} .
$$

Again, although the accuracy of the value $\alpha_{\mathrm{FK} 51}=2.4$. $10^{-3} \mathrm{~cm}^{-1}$ may be not high (see above), it is important to note that the value of the product $\alpha_{\mathrm{FK} 51} P_{\mathrm{FK} 51}$ is measured with high accuracy

$$
\alpha_{\mathrm{FK} 51} P_{\mathrm{FK} 51}=-(0.41 \pm 0.06) 10^{-8} \mathrm{~K}^{-1} \cdot \mathrm{cm}^{-1}
$$

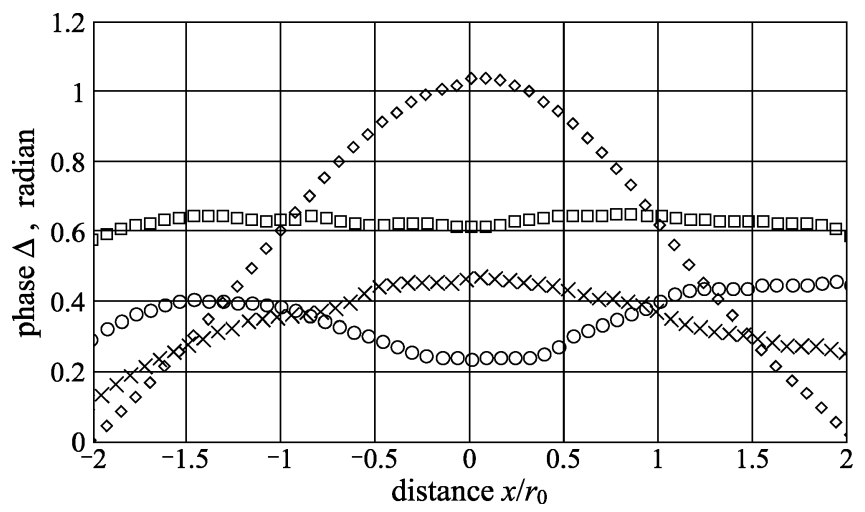

Fig. 7. Probe laser optical path difference after propagating through a birefringence-compensated FI without thermal compensation (diamonds) and with thermal compensation by means of FK51 glass: $\varphi$-polarization (circles), $r$-polarization (crosses), average between two polarizations (squares). The heating laser power is $38 \mathrm{~W}$.

which, considering (33), yields the critical parameter $P / Q$ (see Section II)

$$
\frac{P_{\mathrm{FK} 51}}{Q_{\mathrm{FK} 51}}=2.8 \pm 0.4 .
$$

The accuracy of the measurement does not depend on the uncertainty in the value of $\alpha_{\mathrm{FK} 51}$. It is seen from Fig. 5 that the astigmatism of the thermal lens is considerably higher for FK51 than for TGG, which is a result of lower ratio $P / Q$.

It is worth noting that the measurements of $P$ are made at a wavelength of $815 \mathrm{~nm}$. To verify that $P$ weakly depends on wavelength, we performed measurements at a wavelength of $1060 \mathrm{~nm}$. Since the wavelength of the probe and heating lasers were very close, we used a more sophisticated experimental technique described in [28]. A sample was heated not by a Gaussian but rather a $\Pi$-shaped (flat-top) beam with a diameter equal to the crystal diameter. Corresponding points are shown in Fig. 6. It is evident that the value of $P_{[001]}$ for TGG is the same, within the experimental error, for these wavelengths.

\section{Experimental Determination of Thermal Lens Compensation in FI Using FK51 Glass}

Our scanning Hartmann sensor was also used to verify experimentally the adaptive compensation method [19] on a birefringence-compensated FI [5], [8], [12]. First, a measurement was taken of the radial distribution of the phase for a two-crystal FI (TGG in the [111] orientation) with quartz rotator but without polarizers. Then, based on the data obtained and constants of FK51 glass, we calculated the length of compensating glass that would be optimal for this FI, in this case $45 \mathrm{~mm}$. The glass was placed between the FI and lens (10) (Fig. 3). Between the lens and the CCD camera, a calcite wedge was used to observe separately the vertical and horizontal polarization of the probe laser.

The results of the measurements are presented in Fig. 7, which shows that the thermal lens averaged for two polarizations (shown as squares) is almost totally compensated at a heating power of $38 \mathrm{~W}$ : at $r<\sqrt{2} r_{0}$ (i.e., in the area of $1 / \mathrm{e}^{2}$ by beam intensity) the difference of phase from constant is less than $0.02 \mathrm{rad}$. At the same time, the astigmatism of the resulting lens evident in the $\varphi$-polarization (circles) and $r$-polarization (crosses) is very large, owing to the small ratio $P_{\mathrm{FK} 51} / Q_{\mathrm{FK} 51}$. 


\section{DISCUSSION}

The investigations that we have performed demonstrate that when calculating power losses of radiation in the spatial polarization mode, the photoelastic effect must be taken into account both in the magnetooptical medium and in the compensation glass. It is important to consider the isotropic change of the refractive index, characterized by the second component in (11) for $P_{[001]}$, as well as anisotropic effects (characterized by parameter $Q$ ), which lead to depolarization and amplitude-phase distortions.

First, we compare the relative contributions of the temperature dependence of refractive index and the photoelastic effect to the thermal lens (two components in (11) for $P_{[001]}$ ). The value of $(d n / d T)_{\mathrm{TGG}}$ was measured in [28] and is $19 \cdot 10^{-6} \mathrm{~K}^{-1}$, which coincides with the value $20 \cdot 10^{-6} \mathrm{~K}^{-1}$ reported in [19], [20]. Comparing this value with (35) we can conclude that the contribution of the photoelastic effect into the thermal lens is one third that of $d n / d T$, and signs of these effects are additive. For FK51 the value $(d n / d T)_{\mathrm{FK} 51}=-7.3 \cdot 10^{-6} \mathrm{~K}^{-1}$ is given in the in the Schott glass catalog for $\lambda=1064 \mathrm{~nm}$ and weakly depends on the wavelength. The comparison of this value with (36) allows us to conclude [see the first equation in (11)] that the contribution of the photoelastic effect in the parameter $P$ is roughly 0.75 of the contribution of temperature dependence of the refractive index, and the signs of these effects are different, resulting in a value for $P_{\mathrm{FK} 51} 4$ times less than $(d n / d T)_{\mathrm{FK} 51}$.

Note that in [20] there is a statement that the contribution of the photoelastic effect in the thermal lens for TGG is $17 \%$ of that of $d n / d T$, and their signs are different. This is in fact incorrect, and arises from an incorrect interpretation of the photoelastic effect-[20, eq. (6)] is incorrect. Referring to [20], the authors of [19] (which happen to be some of the same authors of this work), state that in many cases the contribution of the photoelastic effect is negligibly small in comparison with $d n / d T$, which, as follows from the above discussion, is incorrect either for TGG or for FK51.

A stronger thermal lens due to the photoelastic effect in TGG and a weaker thermal lens in FK51 can nevertheless have the same modulus and different signs provided the length of glass is chosen properly (considering the photoelastic effect) (see Fig. 6). However, the anisotropic part of the photoelastic effect in TGG leads to power losses in mode $\gamma_{p}$ and $\gamma_{a}$ that cannot be compensated. Moreover, the anisotropic part of the photoelastic effect induces additional losses. In this regard, the figure of merit of glass is the ratio $P_{\mathrm{CG}} / Q_{\mathrm{CG}}$, which should be maximized for best performance. For the FK51 glass, this ratio is as small as 2.8 , thereby considerably increasing $\mathrm{AC}_{a \mathrm{AC}}$ and $\mathrm{AC}_{p \mathrm{AC}}$, [see (18)]. Fig. 8 shows dependences of different losses plotted by (17), (25), and (27) taking into account the conditions (23) and (24). The dashed lines indicate the dependences obtained by numerically integrating (2) and (3) without the approximation of weak distortions used when deducing (17), (25), and (27). Although there is a small difference between the analytical and numerical results, it is evident that the analytical results fairly well describe $\mathrm{AC}$ in the limit $\mathrm{AC}<0.1$. As one can see from Fig. 8, the telescope method is better than adaptive method with FK51 glass. This comes about because the figure

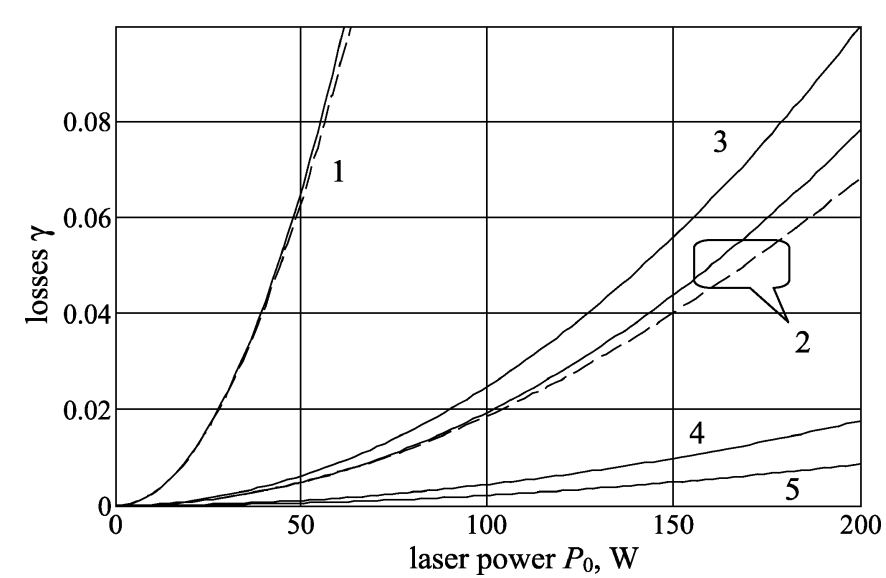

Fig. 8. Theoretically predicted power losses $\gamma$ versus laser power $P_{0}$ for the birefringence-compensated FI shown in Fig. 1(b) for no thermal compensation (curve 1), with telescope compensation (curve 2), adaptive compensation by means of FK51 (curve 3), by means of glass with $P_{\mathrm{CG}} / Q_{\mathrm{CG}}=12$ (curve 4), by means of uniaxial crystal or gel (curve 5). The dashed lines show results of numerical integration and solid lines show analytical results given by (17), (25), (27), and (39). For these calculations, we assumed a total length $L=24 \mathrm{~mm}$ of two TGG crystals, and TGG absorption $\alpha_{\mathrm{TGG}}=2.5 \cdot 10^{-3} \mathrm{~cm}^{-1}$.

of merit $P_{\mathrm{FK} 51} / Q_{\mathrm{FK} 5}=2.8$ is less than 3.2 , the right-hand side of (29b). However, telescopic compensation is a point design for a particular power; values plotted for telescopic compensation in Fig. 8 assume that an optimal compensating focus is achieved at each power, requiring repositioning of the compensating lens. There are two ways of eliminating the negative influence of the anisotropic part of the photoelastic effect in the adaptive compensation method: by compensating the depolarization in the compensating element or by reducing its influence to a negligible level. The depolarization can be compensated using two elements made of FK51 glass and a $90^{\circ}$ polarization rotator placed between them, as is done in active elements of solid-state lasers [33]. If the total length of these two elements provides for condition (23), then the isotropic distortions in the FI will be compensated. This can be called ideal compensation, since, although the anisotropic part remains the same as without the compensating glass, yet

$$
\gamma_{t \mathrm{PC}}=\gamma_{p \mathrm{NC}}+\gamma_{a \mathrm{NC}}
$$

This method, however, has the disadvantage of complexity and losses associated with residual reflections from surfaces of additional elements.

The effect of thermally induced anisotropy of the compensating glass can be considerably reduced by choosing a glass with a large ratio $P_{\mathrm{CG}} / Q_{\mathrm{CG}}$. It is seen from (17), (18), (24)-(26), (31), and (35) that at $P_{\mathrm{CG}} / Q_{\mathrm{CG}}=12$, losses $\gamma_{t \mathrm{AC}}$ during adaptive compensation are only twice as much as $\gamma_{t \mathrm{PC}}$ (see Fig. 8), and at $P_{\mathrm{CG}} / Q_{\mathrm{CG}}>50$, the negative influence of the compensating glass can be neglected in practice. Note, that compensating glass with $Q_{\mathrm{CG}}>0$ would be more preferable because in this case the last term in (18) for $\gamma_{a \mathrm{AC}}$ is negative $(p<0)$. The photoelastic effect can be totally eliminated by using gel as the compensating medium rather than glass, as it is done for compensation of the thermal lens in active laser media [34], or a crystal with natural birefringence, with which 
the induced birefringence can be neglected [23]. An example of such a crystal may be LICAF or YLF. The last one has [35]

$$
\frac{d n}{d T}= \begin{cases}-2 \cdot 10^{-6} \mathrm{~K}^{-1}, & \text { ordinary } \\ -4.3 \cdot 10^{-6} \mathrm{~K}^{-1}, & \text { extraordinary. }\end{cases}
$$

To increase absorption, YLF can be doped by copper, ytterbium, or another element.

Note that in contrast to glass, a gel or an anisotropic crystal can be placed between polarizers of FI, without deteriorating the isolation ratio. This reduces the distance from the gel (or crystal) to the TGG crystal, thereby decreasing diffraction losses [19]. Another important advantage of YLF is that its thermal conductivity $(6 \mathrm{~W} / \mathrm{K} \cdot \mathrm{m}[35])$ and the product of thermal capacity $(790 \mathrm{~J} / \mathrm{kg} \cdot \mathrm{K})$ [35] and density $\left(3.96 \mathrm{~g} / \mathrm{cm}^{3}\right)$ [35] are close to the thermal conductivity and corresponding product of the TGG crystal (thermal conductivity $385 \mathrm{~J} / \mathrm{kg} \cdot \mathrm{K}$, density $7.32 \mathrm{~g} / \mathrm{cm}^{3}$ ) [36]. Due to this, the thermal lens can be compensated not only in the stationary regime, but also dynamically in the presence of rapidly changing beam power. When gel or an anisotropic crystal is used, the total power loss $\gamma_{t}$ is described by (39). The dependence of $\gamma_{t \mathrm{PC}}$ on radiation power is plotted in Fig. 8. Here, it is evident how efficient the adaptive compensation can be.

\section{CONCLUSION}

We have performed a comprehensive investigation of thermally induced self-action of laser beam propagation in TGGbased FIs. The absorption of laser power leads to losses in the initial spatial polarization mode of laser radiation. These losses $\gamma_{t}$ consist of three components: losses induced by isotropic thermal lens $\gamma_{i}$; polarization losses $\gamma_{p}$; and losses associated with amplitude-phase distortions due to depolarization $\gamma_{a}$. The key parameters for determining $\gamma_{t}$ are the thermooptic constants $P$, which determines the isotropic losses $\gamma_{i}$, and $Q$, which determines the anisotropic losses $\gamma_{p}+\gamma_{a}$. We have measured these constants for TGG crystals and FK51 glass and find that for TGG, the contribution of the photoelastic effect to the isotropic component of the thermal lens is comparable in magnitude to the lens induced by $d n / d T$, and, because their contributions are additive, the actual thermal lens is stronger than the lens obtained when the photoelastic effect is neglected. For FK51 glass, the influence of the photoelastic effect is even higher. Thus, the contribution of the photoelastic effect in an isotropic thermal lens should be taken into account when the lens is compensated with an ordinary lens or a telescope with optimal focus [10] or using the adaptive method [19]. In the latter case, the figure of merit of the compensating glass is a ratio of thermooptical constants $P$ and $Q$. For the FK51 glass, the efficiency of the adaptive method is less than for the ordinary lens with an optimal focus, because the value of $P / Q$ for this glass is small. However, the efficiency of the adaptive compensation can be considerably enhanced by eliminating the anisotropy with a $90^{\circ}$ polarization rotator or using a compensating glass with $P_{\mathrm{CG}} / Q_{\mathrm{CG}}>50$, such as a crystal with natural birefringence or a gel.

\section{REFERENCES}

[1] V. Gapontsev and W. Krupke, "Fiber lasers grow in power," Laser Focus World, vol. 38, pp. 83-87, 2002.

[2] Y. Akiyama, H. Takada, H. Yuasa, and N. Nishida, "Efficient $10 \mathrm{~kW}$ diode-pumped Nd:YAG rod laser," presented at the Advanced Solid State Lasers Conf., Quebec City, QC, Canada, 2002.
[3] A. N. Malshakov, G. Pasmanik, and A. K. Potemkin, "Comparative characteristics of magneto-optical materials," Appl. Opt., vol. 36, pp. 6403-6410, 1997.

[4] T. V. Zarubina, A. N. Mal'shakov, G. A. Pasmanik, and A. K. Poteomkin, "Comparative characteristics of magnetooptical glasses," Opticheskii Zh., vol. 64, pp. 67-71, 1997.

[5] E. A. Khazanov, "Compensation of thermally induced polarization distortions in Faraday isolators," Quantum Electron., vol. 29, pp. 59-64, 1999.

[6] E. A. Khazanov, O. V. Kulagin, S. Yoshida, D. Tanner, and D. Reitze, "Investigation of self-induced depolarization of laser radiation in terbium gallium garnet," IEEE J. Quantum Electron., vol. 35, pp. 1116-1122, Aug. 1999.

[7] H. J. Eichler, O. Mehl, and J. Eichler, "Multi-amplifier arrangements with phase conjugation for power scaling of solid state lasers with high beam quality," in Proc. SPIE, vol. 3616, 1999, pp. 166-176.

[8] E. Khazanov, N. Andreev, A. Babin, A. Kiselev, O. Palashov, and D. Reitze, "Suppression of self-induced depolarization of high-power laser radiation in glass-based Faraday isolators," J. Opt. Soc. Amer. B, Opt. Phys., vol. 17, pp. 99-102, 2000.

[9] N. Andreev, A. Babin, A. Kiselev, O. Palashov, E. Khazanov, O. Shaveleov, and T. Zarubina, "Thermooptical constant of magneto-active glasses," J. Opt. Technol., vol. 67, pp. 556-558, 2000.

[10] E. A. Khazanov, "Characteristic features of the operation of different designs of the Faraday isolator for high average laser-radiation power," Quantum Electron., vol. 30, pp. 147-151, 2000.

[11] - "High-power propagation effects in different designs of a Faraday isolator," presented at the Optical Pulse and Beam Propagation II Conf., San Jose, CA, 2000

[12] N. F. Andreev, O. V. Palashov, A. K. Poteomkin, A. M. Sergeev, E. A. Khazanov, and D. H. Reitze, "45 dB Faraday isolator for $100 \mathrm{~W}$ average radiation power," Quantum Electron., vol. 30, pp. 1107-1108, 2000.

[13] K. S. Lai, R. Wu, and P. B. Phua, "Multiwatt $\mathrm{KTiOPO}_{4}$ optical parametric oscillators pumped within randomly and linearly polarized Nd:YAG laser cavities," presented at the Nonlinear Materials, Devices and Applications Conf., San Jose, CA, 2000.

[14] E. Khazanov, N. Andreev, O. Palashov, A. Poteomkin, A. Sergeev, O. Mehl, and D. Reitze, "Effect of terbium gallium garnet crystal orientation on the isolation ratio of a Faraday isolator at high average power," Appl. Opt., vol. 41, pp. 483-492, 2002.

[15] N. F. Andreev, E. V. Katin, O. V. Palashov, A. K. Potemkin, D. Reitze, A. M. Sergeev, and E. A. Khazanov, "The use of crystalline quartz for compensation for thermally indused depolarization in Faraday isolators," Quantum Electron., vol. 32, pp. 91-94, 2002.

[16] M. R. Ostermeyer, G. Klemz, P. Kubina, and R. Menzel, "Quasicontinuous-wave birefringence-compensted single- and double-rod Nd:YAG lasers," Appl. Opt., vol. 41, pp. 7573-7582, 2002.

[17] K. A. Strain, K. Danzmann, J. Muzino, P. G. Nelson, A. Rudiger, R. S. Schillng, and W. Winkler, "Thermal lensing in recycling interferometric gravitational-wave detectors," Phys. Lett. A, vol. 194, pp. 124-132, 1994.

[18] C. A. Denman and S. I. Libby, "Birefringence compensation using a single Nd:YAG rod," in OSA TOPS'99, vol. 26, 1999, pp. 608-612.

[19] G. Mueller, R. S. Amin, D. Guagliardo, D. McFeron, R. Lundock, D. H. Reitze, and D. B. Tanner, "Method for compensation of thermally induced modal distortions in the input optical components of gravitational wave interferometers," Classical Quantum Gravity, vol. 19, pp. 1793-1801, 2002.

[20] J. D. Mansell, J. Hennawi, E. K. Gustafson, M. M. Fejer, R. L. Byer, D. Clubley, S. Yoshida, and D. H. Reitze, "Evaluating the effect of transmissive optic thermal lensing on laser beam quality with a Shack-Hartmann wave-front sensor," Appl. Opt., vol. 40, pp. 366-374, 2001.

[21] A. P. Voytovich and V. N. Severikov, Lasers With Anisotropic Resonators. Minsk, Belarus: Nauka i Tehnika, 1988.

[22] M. J. Tabor and F. S. Chen, "Electromagnetic propagation through materials possessing both Faraday rotation and birefringence: Experiments with ytterbium orthoferrite," Appl. Phys., vol. 40, pp. 2760-2765, 1969.

[23] A. V. Mezenov, L. N. Soms, and A. I. Stepanov, Thermooptics of SolidState Lasers. $\quad$ Leningrad, U.S.S.R.: Mashinostroenie, 1986.

[24] W. Koechner, "Thermal lensing in a Nd: YAG laser rod," Appl. Opt., vol. 9, pp. 2548-2553, 1970.

[25] A. Anan'ev, N. A. Kozlov, A. A. Mak, and A. I. Stepanov, "Thermal distortion of solid state laser cavity," Prikl. Spektroskopiya, vol. 5, pp. 51-55, 1966.

[26] L. N. Soms and A. A. Tarasov, "Thermal deformation in color-center laser active elements. 1. Theoty," Sov. J. Quantum Electron., vol. 9, pp. 1506-1508, 1979. 
[27] A. A. Mak, L. N. Soms, V. A. Fromzel, and V. E. Yashin, Nd:glass Lasers. Moscow, Russia: Nauka, 1990.

[28] A. Poteomkin, N. Andreev, E. Khazanov, A. Shaykin, V. Zelenogorsky, and I. Ivanov, "Use of scanning Hartmann sensor for measurement of thermal lensing in TGG crystal," presented at the Laser Crystals, Glasses, and Nonlinear Materials Growth and Characterization III Conf., San Jose, CA, 2003.

[29] A. Anan'ev and N. I. Grishmanova, "Deformation of active elements of interferometer and thermooptical constant Nd: glass," Prikl. Spektroskopiya, vol. 12, pp. 668-673, 1970.

[30] A. A. Mak, V. M. Mit'kin, and L. N. Soms, "About thermooptical constant of doped glasses," Opt.-Mekh. Prom., vol. 9, pp. 65-66, 1971.

[31] M. Born and E. Wolf, Principles of Optics. New York: Pergamon, 1980.

[32] M. Abitbol, X. Ben-Yosef, and N. Nissim, "Use of the Hartmann sensor to measure the unisoplanatic wavefront tilt," Appl. Opt., vol. 30, pp. $1512-1523,1991$

[33] W. C. Scott and M. de Wit, "Birefringence compensation and Tem00 mode enhancement in a Nd:YAG laser," Appl. Phys. Lett., vol. 18, pp. 3-4, 1971.

[34] T. Graf, E. Wyss, M. Roth, and H. P. Weber, "Adaptive thermal optics in high-power laser resonators," presented at the SPIE Laser Resonators and Beam Control Conf., San Jose, CA, 2002.

[35] G. M. Zverev and D. Golyaev, Crystaline Lasers and Its Application. Moscow, Russia: Radio and sviaz, 1994.

[36] T. V. Zarubina, private communication, Mar. 2003.

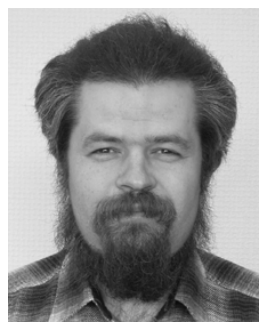

Efim Khazanov was born in 1965 in Gorky (now Nizhny Novgorod), U.S.S.R. (now Russia). He received the Ph.D. degree in physics and mathematics from the Institute of Applied Physics, Russian Academy of Sciences, Nizhny Novgorod, Russia, in 1992.

His research is in the field of phase conjugation of depolarized radiation, stable narrow bandwidth $Q$-switch lasers, diffraction-limited solid-state lasers with high peak and average power, thermo-optics of solid-state lasers, and optical parametrical amplification of chirped pulse. He is currently a Department Head in the Institute of Applied Physics, Russian Academy of Sciences, and an Assistant Professor at Nizhny Novgorod State University. He is the author of more than 100 publications.

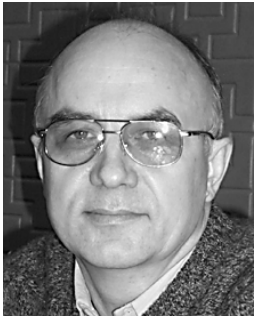

Nikolay F. Andreev was born in Chkalovsk, Gorky (now Nizhny Novgorod) Region, U.S.S.R. (now Russia), in 1947. He received the B.S. degree in radiophysics, specializing in the field of nonlinear optics, specifically on problems of stimulated scattering of light and adaptive optics, and the Ph.D. degree in physics and mathematics from Gorky State University, Gorky, U.S.S.R., in 1973 and 1984, respectively.

He is currently Head of the Laboratory of Pulsed Solid-State Lasers at the Institute of Applied Physics, Russian Academy of Sciences, Nizhny Novgorod, Russia, where he investigates problems in applied laser physics.

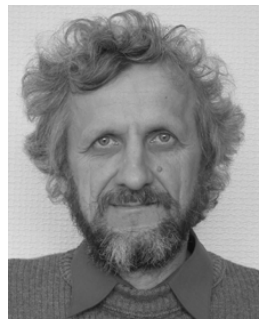

Anatoly Mal'shakov was born in 1950 in the Kirov Region, U.S.S.R. He received the M.D. degree in physics from Gorky State University, Gorky (now Nizhny Novgorod), U.S.S.R. (now Russia), in 1976.

His initial research was in the field of physics of metals. Since 1984, he has worked in the area of laser physics at the Institute Applied Physics, Russian Academy of Sciences, Nizhny Novgorod, Russia.

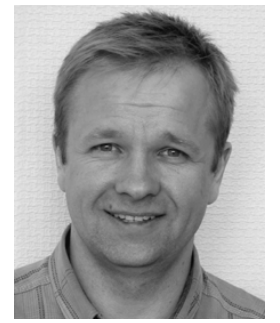

Oleg Palashov was born in Gorky (now Nizhny Novgorod), U.S.S.R. (now Russia), in 1966. He received the Ph.D. degree in physics and mathematics from the Institute of Applied Physics, Russian Academy of Sciences, Nizhny Novgorod, Russia, in 1999.

$\mathrm{He}$ is currently with the Institute of Applied Physics, Russian Academy of Sciences. His research is in the field of phase conjugation of depolarized radiation, stable narrow bandwidth $Q$-switch lasers, diffraction-limited solid-state lasers with high peak and average power, and thermo-optics of solid-state lasers. $\mathrm{He}$ is the author of more than 60 publications.

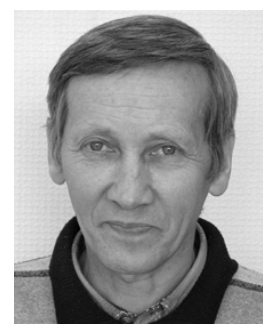

Anatoly K. Poteomkin was born in Gorky (now Nizhny Novgorod), U.S.S.R. (now Russia), in 1949. He graduated from Gorky State University, Gorky, U.S.S.R., in 1973.

$\mathrm{He}$ is currently a Senior Scientist at the Institute of Applied Physics, Russian Academy of Sciences, Nizhny Novgorod, Russia. His research is in the fields of laser engineering, nonlinear optics, problems of stimulated scattering of light, self-focusing, high-efficiency generation of laser harmonics, precision measurement of light-wave fronts, and adaptive optics. He has authored more than 70 publications.

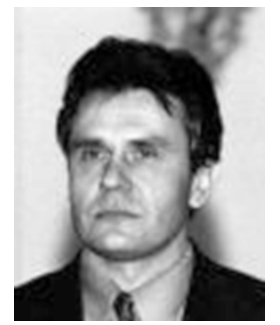

Alexander Sergeev graduated from Gorky State University, Gorky (now Nizhny Novgorod), U.S.S.R. (now Russia), in 1977.

Since that time, he has been with the research staff of the Institute of Applied Physics of the Russian Academy of Sciences (IAP RAS), Nizhny Novgorod, Russia. Currently, he is the Director of the Division for Nonlinear Dynamics and Optics and the Deputy-Director of IAP RAS. His main activities are related to femtosecond optics, development of high-power lasers, interaction of intense radiation with matter, laser-biotissue interactions, and femtosecond spectroscopy and tomography. He has contributed to more than 150 scientific publications.

Mr. Sergeev was the winner of the Russian State Prize in 1999 for pioneering work in optical coherence tomography. In 2003, he was elected a Corresponding Member of the Russian Academy of Sciences.

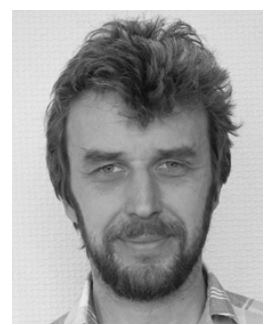

Andrey A. Shaykin was born in the Nizgny Novgorod Region, U.S.S.R., in 1965. He received the $\mathrm{Ph} . \mathrm{D}$. degree in physics and mathematics from the Institute of Applied Physics, Russian Academy of Sciences, Nizhny Novgorod, Russia, in 2001. His $\mathrm{Ph} . \mathrm{D}$. dissertation was devoted to excitation and propagation of low and upper hybrid electromagnetic waves in ionospheric plasma in nonuniform density ducts and nonlinear effects near antennas in magnetoplasma.

$\mathrm{He}$ is currently with the Institute of Applied Physics, Russian Academy of Sciences. His current research is in field of high power and high energy solid-state lasers and optics.

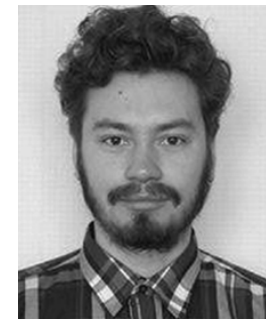

Victor Zelenogorsky was born in 1978. He graduated from the Institute of Applied Physics, Lobachevsky University of Nizhny Novgorod, Russia, in 2001.

Since 2001, he has been with the Institute of Applied Physics, Russian Academy of Sciences, specializing in thermo-optics and solid-state lasers. He has authored ten papers published in journals. 


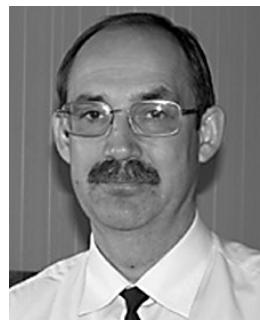

Igor A. Ivanov was born in Moscow, U.S.S.R., in 1955. He received the electronic engineer diploma from Moscow Institute of Electronic Technology, Moscow, U.S.S.R., in 1978, and the Ph.D. degree from the Research Institute of Material Science and Technology, Zelenograd, Russia, in 1988. His dissertation was a study of growth of large-sized gadolinium gallium garnet crystals for bubble memory.

Recently, he joined the Research Institute of Materials Science and Technology, Zelenograd, Russia, where he works on development of optical and laser garnet crystal growth.

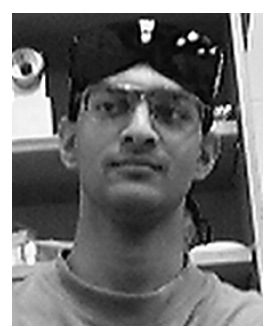

Rupal Amin was born in Leeds, U.K., in 1977. He received the B.S. degree (with honors) in physics from Florida Institute of Technology (Florida Tech), Melbourne, in 1999.

At Florida Tech, he worked in several physics laboratories mentored by Dr. R. Raffallee, Dr. H. K. Rassoul, and Dr. M. Moldwin. He also obtained his first experience with NASA and extended duration microgravity experiments. After graduation, he joined the University of Florida, Gainesville, where he worked with Dr. D. B. Tanner and Dr. D. Reitze. Under their guidance, he entered the LIGO project and began working with Dr. G Mueller on high-power experiments. These experiments lead to his joining the LIGO Livingston Observatory where he is currently establishing the new University of Florida High Power Laser Facility for Advanced LIGO.

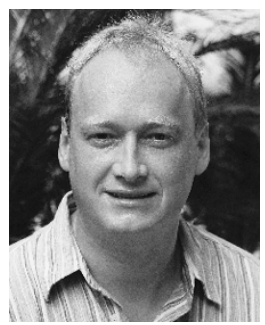

Guido Mueller is a native of in Porta Westfalica, Germany. He received the diploma from the Institute of Quantum Optics, University of Hannover, Hannover, Germany, in 1993, and the Ph.D. degree from the Institute of Atomic and Molecular Physics, University of Hannover, in 1997.

Upon graduation, he became a JSPS Postdoctoral Fellow at the Institute for Laser Science at the University of Electro-Communication, Tokyo, Japan, in 1997, followed by positions as a Postdoctoral Research Associate (1998-2000) and later a Research Scientist (2000-2003) in the LIGO group at the University of Florida, Gainesville. In 2002 and 2003, he also worked in the LISA Laboratory at the Goddard Space Flight Center. Since August 2003, he has been an Assistant Professor in the Department of Physics, University of Florida. He specializes in laser interferometry and material research for interferometric gravitational wave detectors (LIGO, LISA).

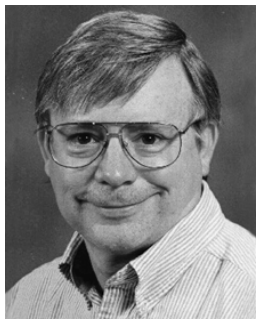

David B. Tanner received the B.A. and M.S. degrees from the University of Virginia, Charlottesville, in 1966 and 1967, respectively, and the Ph.D. degree from Cornell University, Ithaca, NY, in 1972.

He held a postdoctoral position for two years at The University of Pennsylvania and then, in 1974, joined the Physics Department at The Ohio State University, where he was an Assistant Professor and Associate Professor. He joined the University of Florida, Gainesville, in 1982, where he is currently Distinguished Professor of Physics. He served as Chair of the Department during 1986-1989. His research is in the area of the optical properties of solids, with emphasis on high-temperature superconductors, lowdimensional materials, polymers, and thin films. A second research area is particle-astrophysics, including gravitational-wave detection and the search for axions, a possible component of the dark-matter halo of our galaxy. His research has been supported by the National Science Foundation, U.S. Department of Energy, and Defense Advanced Research Projects Agency. He is the author of more than 300 scientific papers and has supervised 20 Ph.D. and nine M.S. students.

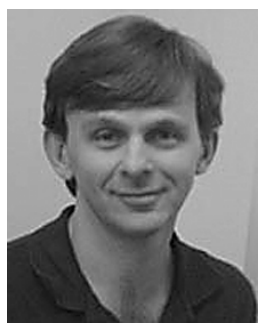

David H. Reitze was born in Pittsburgh, PA, in 1961. He received the B.A. degree from Northwestern University, Chicago, IL, in 1983, and the Ph.D. from the University of Texas, Austin, in 1990, both in physics.

From 1990 to 1992, he was a Postdoctoral Member of Technical Staff in the Ultrafast Optics and Optical Signal Processing Group, Bell Communications Research, Red Bank, NJ. In 1992, he joined Lawrence Livermore National Laboratory, Livermore, CA. Since 1993, he has been with the University of Florida, Gainesville, where he is currently a Professor of Physics. His research interests lie in the areas of ultrafast lasers and spectroscopy and interferometric gravitational-wave detection. He is the author of over 80 scientific publications.

Prof. Reitze has served on numerous conference program committees for the optics community. 